\title{
A SEIQR Model considering the Effects of Different Quarantined Rates on Worm Propagation in Mobile Internet
}

\author{
Yonghua Zheng, ${ }^{1,2}$ Jianhua Zhu ${ }^{1}{ }^{1}$ and Chaoan Lai ${ }^{1}$ \\ ${ }^{1}$ School of Business Administration, South China University of Technology, Guangzhou 510641, China \\ ${ }^{2}$ Guizhou MinZu University, Guiyang, China \\ Correspondence should be addressed to Jianhua Zhu; 201710105331@mail.scut.edu.cn
}

Received 18 December 2019; Revised 19 March 2020; Accepted 8 April 2020; Published 26 May 2020

Academic Editor: Libor Pekař

Copyright (C) 2020 Yonghua Zheng et al. This is an open access article distributed under the Creative Commons Attribution License, which permits unrestricted use, distribution, and reproduction in any medium, provided the original work is properly cited.

\begin{abstract}
At present, $\mathrm{Wi}-\mathrm{Fi}$ is a common medium for connecting smart devices to networks in factories. The application of mobile Internet in smart manufacturing system (SMIS) speeds up the process of smart manufacturing but also increases SMIS vulnerability to worm attack from mobile networks. In this paper, we propose a new SLBQR (susceptible-latent-breaking out-quarantinedrecovered) model considering vaccination strategies with temporary immunity and quarantined strategies. Based on basic reproduction number, we give expression of quarantined rate $\varphi$ and obtain the threshold $\phi^{*}$ of quarantined rate $\varphi$ such that the worm-free equilibrium is asymptotically stable when $\phi \geq \phi^{*}$, implying that the worm dies out eventually and its attack remains under control; the endemic equilibrium is asymptotically stable when $\varphi<\varphi^{*}$, namely, the worm is always persistent and spreading within a population. Hence, we give the quarantined strategy $\phi \geq \phi^{*}$ to suppress the spread of virus. In addition, by theoretical analysis, we can conclude that even if the immunity time is unlimited, endemic equilibrium will not become worm-free. In other words, there is a mutation in the virus, which proves that there is no vaccination strategy with permanent immunity. Finally, we simulate our model with different temporary immune time and quarantine rates, and the results verify our theorem.
\end{abstract}

\section{Introduction}

Today's world is at the beginning of the period of communication and universal processing. In this period, society is guided towards the always connected pattern. With the introduction of new technologies, the coverage of networks has been widened and the number of smart objects that are connected to the network has also been increased. A new pattern called the Internet of things (IoT) increases the amount of information generated on the Internet through connecting people and smart objects [1]. In industrial production, the connection mode between smart objects is through wireless sensor network, and the connection between smart objects and people is through the SMIS. Although, the new generation of IoT has been created to achieve quality of service (QoS), in practical industrial applications, the two connection modes of wireless sensor networks and information systems face many challenges. In the research of wireless sensor networks, the limited energy of wireless sensors is a big challenge of wireless sensor networks; hence, resource allocation and optimization is the hotspot and difficulty of research. In this research, Kumar Sangaiah et al. [1] used the total communication cost minimum as the objective function and used whale optimization algorithm (WOA) to solve the RA problem and reduce the total communication cost between resources and gateways. Sangaiah et al. [2] used bat algorithm (BA) to select the optimum monitoring sensor node and resulted path to extend the network lifetime through extending the lifetime of operating sensors as well transferring gathered data from supernode to the sink.

Since the manufacturing equipment is fixed, the connection between the smart objects through the wireless sensor network is in a relatively fixed environment. However, due to the flexibility of the operator, the connection between smart objects and people is in a changing 
environment. Therefore, compared with the wireless sensor network used for the connection of things, the information system used for the connection of people and things is more vulnerable, and once the manufacturing information system fails, it will bring greater disaster to the manufacturing enterprise. In addition, the application of IoT [3] enables enterprises to accomplish their tasks in a mobile way through mobile devices. For example, based on the mobile Internet, employees of manufacturing enterprises can monitor the production status at the time and place they expect, process orders, release work updates, read logs, review financial statements, and so on. With the increase of mobile network in industrial application, the scale of SMIS continues to expand. On the one hand, the growth of mobile devices and the rapid growth of applications installed on these devices have allowed the mobile Internet to scale up SMIS by adding data, mobile devices, and applications. On the other hand, the IoT enables SMIS and its subsystems to run in more places with less time constraints, thus expanding the scale of SMIS. Therefore, the application of IoT makes the traditional manufacturing information systems into smart manufacturing information system. Although it greatly promotes the development of manufacturing enterprises, it also exposes the information system to the threat of cyberattacks. Therefore, from the above analysis, we can find that it is very necessary to study the security of SMIS under cyberattacks.

Cyberattacks' threat to SMIS is mainly reflected in information security and equipment security. Among them, information security is mainly reflected in the communication link layer. In this aspect of research, Sangaiah et al. [4] focused on aspects of conserving position and information confidentiality and proposed an energy-aware green adversary model for its use in smart industrial environment through achieving confidentiality. In addition, traditional information systems rely on stationary workstations, which can only operate in a fixed position. The SMIS makes the operation of the system that has the characteristics of mobility, ubiquity, timeliness, and convenience [5] as shown in Figure 1, so position-based confidentiality becomes a problem that cannot be ignored; in order to solve this problem, Sangaiah et al. [6] proposed machine learning techniques for conserving position confidentiality of roaming PBS users and further used mobile edge computing to offer position confidentiality and low latency by means of networking and computing services at the vicinity of roaming users.

The above information security of SMIS is based on the assumption that the communication equipment is in a healthy state. However, with the development of the IoT technology, Wi-Fi makes it one of the most widely used Internet connections between mobile devices in the industry. However, the application of mobile Internet exposes the smart devices to the dangerous environment. In mobile Internet environment, the biggest threat of these devices is computer worms as shown in Figure 2. Worms are independent programs that run without the intervention of a computer user. They spread by constantly gaining some or all control over computers with holes in the network and can

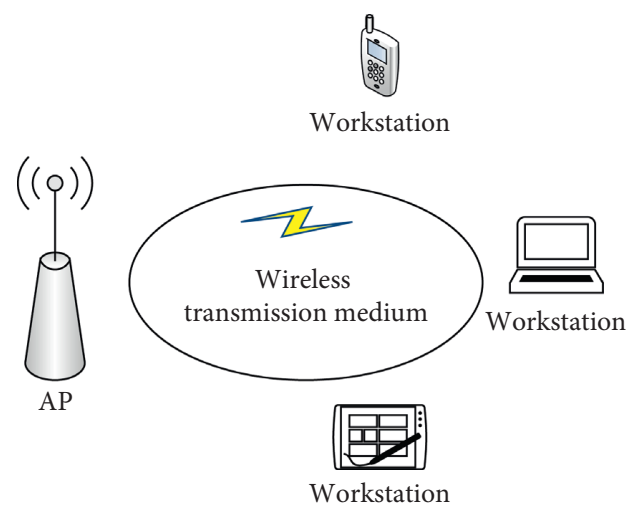

FIgure 1: Mobile internet in industry.

cause network congestion [7]. Most mobile devices in manufacturing companies do not have any effective way to prevent worm attacks, which makes them vulnerable to such attacks. Mobile devices infected by the worm can cause huge losses to manufacturers, including corporate strategy leaks, information system disruptions, and financial losses. Common computer worms include the Code Red worm [8], the Witty worm [9], and the Slammer worm [10]. Therefore, our research of worm attack of SMIS in Wi-Fi environment is very important to the research of SMIS equipment security. This is also the basis of information security.

The research on the attack of worms on equipment of SMIS mainly focuses on the problem of virus transmission between equipment. Over the past few decades, researchers have come up with many different mathematical models to analyze the dynamic behavior and characteristics of worm propagation. The modeling of computer viruses was started by Kephart and White in 1991 [11]. In the follow-up studies, scholars used a series of biological virus transmission models to conduct studies. Martin et al. [12] applied the SIS model to study the impact of mobile malware on cell phones. Feng et al. [13] proposed a time-delayed SIRS model which introduced two parameters: temporal immunity and variable infection rate, and explored the impact of the variable infection rate on the scale of malware outbreak. However, it is unreasonable for the above models to assume that susceptible nodes will immediately become infected nodes. In fact, it takes a certain amount of time for infected nodes to send copies of the worm to susceptible nodes. Therefore, in order to overcome the above deficiency, some researchers introduced exposure status $(E)$ in the model and proposed some new models. Signes-Pont et al. [14] took propagation dynamics of worms in smart phones as example, proposed susceptible-exposed-infected-diagnosed-recovered

(SEIDR). Xia et al. [15] built a theoretical model based on five compartments susceptible-exposed-infected-recovereddormancy (SEIRD) for the Bluetooth and MMS hybrid spread mode. Jia and Jing [16] considered immune time and established SEIRS model with time delay.

Different from above models, in the Wi-Fi environment, base station (BS) can block worm propagation by disconnecting the mobile device from the mobile network. That is, BS can quarantine infected nodes. An important 


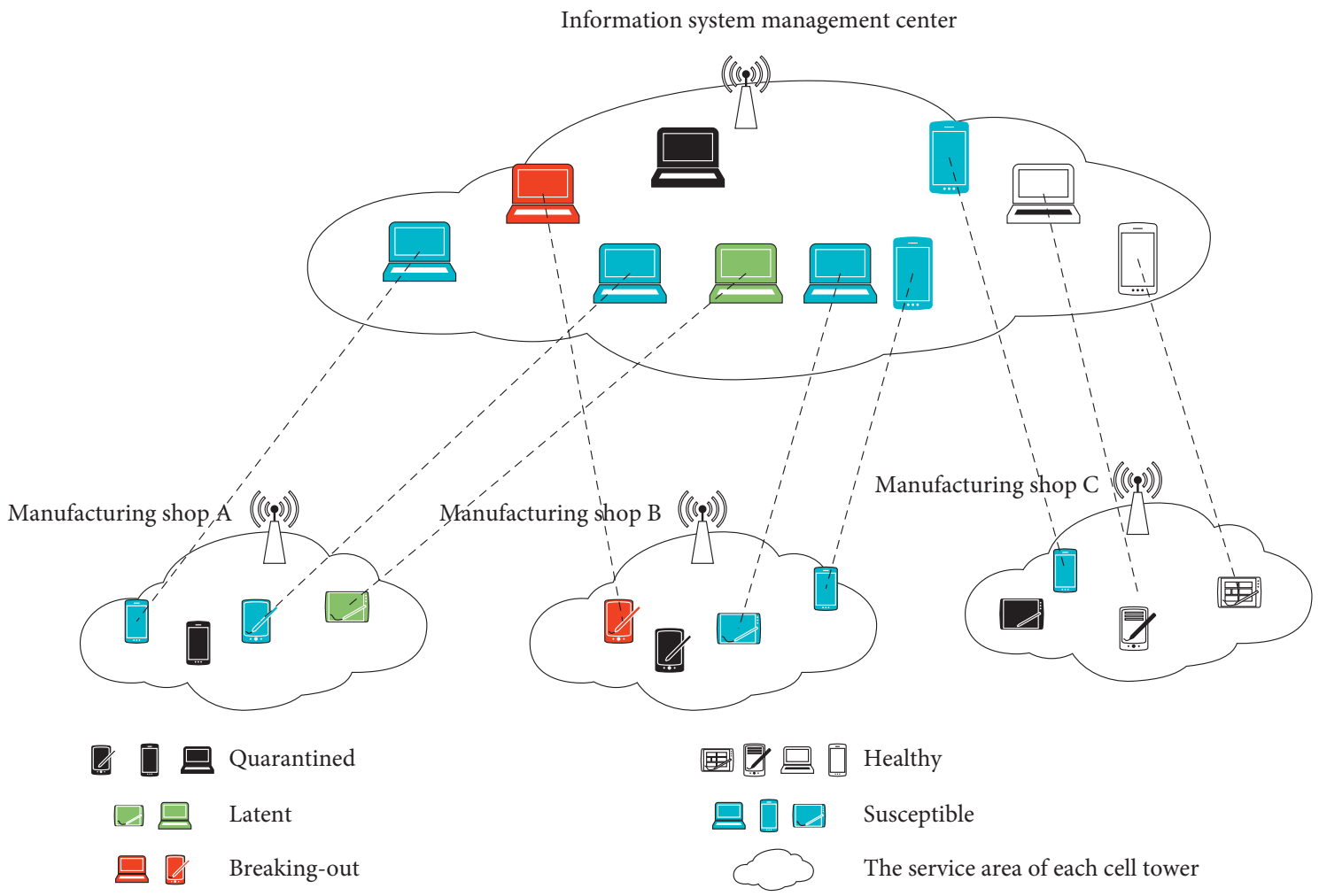

Figure 2: Virus propagation path in the mobile network.

quarantine strategy was taken into account in the DQ model [17], PWDQ model [18], SEIQV model [19], SEIQRS model [20], and SEIQR model [21]. Mishra and Jha [22] proposed a susceptible-exposed-infectious-quarantined-recovered (SEIQR) model for the transmission of malicious objects in computer network. Wang et al. [23] proposed a novel epidemic model which combines both vaccinations and dynamic quarantine methods (SEIQV). Due to the high similarity between network worms and biological viruses, vaccination by installing efficient antivirus software or immunization procedures has been an effective defense mechanism to control the attack of worms, and the vaccination is assumed to have full efficacy and permanent immunity $[24,25]$.

Through the analysis of the above literature, we find that the application of Wi-Fi has caused scholars to widely consider the impact of the quarantined state on the spread of worms between information system equipment. However, the existing research has the following problems:

(1) They only introduce the quarantined state and do not further consider the impact of different quarantined strategies on the spread of worms and what kind of quarantined strategy is adopted to completely suppress the spread of the virus.

(2) Although the vaccination strategy is considered, the vaccination strategy is assumed to have full efficacy and permanent immunity, but in reality, the efficacy of antivirus software does not reach $100 \%$.
In order to solve the shortcomings of the existing research, this paper considers how to obtain the expression of the quarantined rate under the vaccination strategy with temporary immunity and how to obtain the threshold of the quarantined rate through the analysis of worm transmission and then gives quarantined strategies to suppress the spread of viruses.

Our research on the above issues has important academic value for subsequent scholars to study such issues. First of all, our research proves that there is no vaccination strategy for permanent immunity. Secondly, the application of Wi-Fi makes the quarantined state have an important impact on the propagation of worms among information system equipment. Therefore, a quarantined strategy that suppresses the spread of viruses can help manufacturers to isolate equipment reasonably and reduce economic losses. Finally, our research provides an important research direction for the follow-up research: how to give the optimal strategy of quarantined under consideration of the minimum economic loss of the enterprise.

Inspired by the above references, we propose a new SLBQR (susceptible-latent-breaking out-quarantined-recovered) model which focuses on the dynamics of the worm propagation and the quarantined strategies for worm attack. Firstly, based on basic reproduction number, we give expression of quarantined rate, and then by deriving the local and global stabilities in both the worm-free and endemic equilibrium, we prove the worm-free equilibrium is locally and globally asymptotically stable if $\phi \geq((\sigma(1-v)(1-$ $\left.\mu) \bowtie N) /\left(R_{0}(\mu+(1-v)(1-\mu) \emptyset+v(1-\mu)-\sigma(1-\mu) N)\right)\right)-$ 
$(\mu /(1-t \mu))$, whereas the endemic equilibrium is locally asymptotically stable if $\varphi<\left((\sigma(1-v)(1-\mu) \omega N) /\left(R_{0}(\mu+\right.\right.$ $(1-v)(1-\mu) \omega+v(1-\mu)-\sigma(1-\mu) N)))-(\mu /(1-\mu))$. Hence, we give the threshold $\varphi^{*}=\left((\sigma(1-v)(1-\mu) \bowtie N) /\left(R_{0}(\mu+\right.\right.$ $(1-v)(1-\mu) \omega+v(1-\mu)-\sigma(1-\mu) N)))-(\mu /(1-\mu)) \quad$ of quarantined rate and quarantined strategies for the worm attacks. Meanwhile, some numerical simulations are carried out to illustrate the feasibility of our theoretical results and the effectiveness of quarantined strategies. Novelty and contribution of the study are as follows:

(1) We consider a vaccination strategy with temporary immunity. Through theoretical analysis and simulation experiments, it is concluded that even if the immunity time is unlimited, endemic equilibrium will not become worm-free. In other words, there is a mutation in the virus, which proves that there is no vaccination strategy with permanent immunity.

(2) Based on basic reproduction number, we give expression of quarantined rate, give the threshold of the quarantined rate to the spread of virus, and then give the quarantined strategy to suppress the spread of virus.

The rest of this paper is arranged as follows. In Section 2, we establish SEIQR model considering time delay and quarantined rate and obtain specific expression of quarantined rate. In Section 3, we give the effects of different quarantined rates on worm propagation stability and get quarantined policy to block worm propagation. In Section 4, we use numerical simulations to verify our results. In the end, the paper is summarized and discussed with some future works.

\section{SLBQR Model}

Recently, Liu and Yang [19] defined the quarantined status (Q) to represent the infected nodes quarantined by the BS and then established the SEIQR model. This model can describe the dynamic behaviors and characteristics of the worm spreading in a Wi-Fi environment. But in practice, the scenario described in the SEIQR model does not coincide with a real mobile network. Particularly, there are two problematical issues.

Firstly, the recovered mobile device is considered to have permanent immunity. This assumption is very unrealistic. Generally speaking, when a mobile device is affected by worm virus, the corresponding antivirus software for a certain type of worm will make the devices gain immunity. But as time goes on, the worm will mutate, so the antivirus software will fail. Therefore, the immunity obtained by the mobile device is temporary immunity, not permanent immunity. Secondly, we just consider quarantined measures and do not consider vaccination measures. In fact, besides the quarantine strategy, the vaccination strategy with antivirus treatment is usually employed to protect from the worm propagation among mobile devices and decrease the intensity of worm attacks in mobile networks. Also, the effects of other control strategies are analyzed on the latency and breaking-out status to prevent worm propagation in a short period immediately after detecting the worm attack, which greatly mitigates the possibility of worm outbreak in early stage among mobile devices.

The arguments above motivate us to develop a more suitable SLBQR model, where we take temporary immunity, vaccination, and quarantined strategies into account for mobile devices and propose control strategy to prevent the worm propagation in mobile networks. The status of mobile devices is defined as follows: susceptible status $(S)$ includes those devices which are vulnerable to worm attacks in mobile networks; latency status $(L)$ includes those devices which are exposed to the attacks and have been infected, but not actively infectious due to the latent time requirement; breaking-out status $(B)$ includes those exposed devices which are actively searching and targeting new victims; quarantined status $(Q)$ includes those infectious devices which are quarantined by the $B S$ station; recovered status $(R)$ includes those devices which gained one or more security countermeasures, providing the devices with a temporary immunity against the malicious worms. Obviously, $S(t)+L(t)+B(t)+Q(t)+R(t)=N(t)$. The block diagram of SLBQS model status transition and model parameters involved are shown in Figure 3. Table 1 shows notation used in this paper and baseline parameter. Table 2 shows a summary of the status transitions and rates of devices in the SLBQR model. Combining Figure 3 and Tables 1 and 2, the status transitions of devices in SLBQR model are detailed as follows.

The basic assumptions in concern with our model are presented below.

(1) All newly connected computers and mobile devices are all virus free.

(2) External computers and mobile devices are connected to the Internet at positive constant rate $\mu$. Also, internal computers and mobile devices are disconnected from the Internet at the same rate $\mu$.

(3) The infected computers and mobile devices are divided into two statuses: latent status and breaking-out status.

(4) Each virus-free computer and mobile device gets in contact with an infected computer or mobile device at a bilinear incidence rate $\sigma$, where $\sigma$ is positive constant.

(5) Latent computers and mobile devices are recovered by vaccination antivirus software at non-negative constant rate $v$.

(6) The virus breaking-out rate in latent computers and mobile devices is non-negative constant rate $\omega$.

(7) The quarantined rate in breaking-out computers and mobile devices is non-negative constant rate $\phi$.

(8) By using antivirus software and system reinstallation on the quarantined devices, the recovered rate is non-negative constant $\psi$.

(9) Recovered computers and mobile devices can gain temporarily immune period $\tau$, where $\tau$ is constant. 


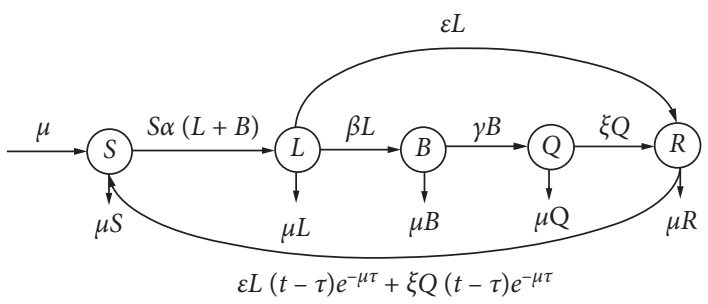

FIGURE 3: Status transition graph of the SLBQR model.

TABLE 1: Notation and parameter values in the model.

\begin{tabular}{lc}
\hline Notation & Explanation \\
\hline$N(t)$ & Total population size \\
$I(t)$ & Infected population \\
$S(t)$ & Susceptible population \\
$L(t)$ & Latent population \\
$B(t)$ & Breaking-out population \\
$Q(t)$ & Quarantined population \\
$R(t)$ & Recovered population \\
$\alpha$ & Status transition rate from $S$ to $L$ \\
$\beta$ & Status transition rate from $L$ to $B$ \\
$\gamma$ & Status transition rate from $B$ to $Q$ \\
$\xi$ & Status transition rate from $Q$ to $R$ \\
$\varepsilon$ & Stats transition rate from $L$ to $R$ \\
$\mu$ & The natural birth rate and the death rate \\
$\tau$ & The temporary immune time from recovered status to suspected status \\
$\omega$ & Worm breaking-out rate \\
$v$ & Vaccination effective rate \\
$\sigma$ & The worm virus infection rate \\
$\phi$ & Quarantined rate \\
$\psi$ & Recovery rate after quarantined \\
\hline
\end{tabular}

TABLE 2: Status transition events and transition rates.

\begin{tabular}{lc}
\hline Events & Rate of transition \\
\hline$S \longrightarrow L$ & $S \alpha(L+B)$ \\
$L \longrightarrow B$ & $\beta L$ \\
$L \longrightarrow R$ & $\varepsilon L$ \\
$B \longrightarrow Q$ & $\gamma B$ \\
$Q \longrightarrow R$ & $\xi Q$ \\
$R \longrightarrow S$ & $\varepsilon L(t-\tau) e^{-\mu \tau}+\xi Q(t-\tau) e^{-\mu \tau}$ \\
$S \longrightarrow$ Dysfunctional & $\mu S$ \\
$L \longrightarrow$ Dysfunctional & $\mu L$ \\
$B \longrightarrow$ Dysfunctional & $\mu B$ \\
$Q \longrightarrow$ Dysfunctional & $\mu Q$ \\
$R \longrightarrow$ Dysfunctional & $\mu R$ \\
Safety $\longrightarrow S$ & $\mu N$ \\
\hline
\end{tabular}

(10) Immune time in the infective including latent status and breaking-out status is exponentially distributed.

The details of the SLBQR model are as follows:

(1) The application of mobile networks in the intelligent manufacturing process exposes some mobile devices to worms and turns them into susceptible status. Worm attack makes $\alpha S(L+B)(\alpha=\sigma(1-\mu))$ devices move form $S$-status to the $L$-status, where ( $1-$ $\mu)$ denotes the normal function rate, $\sigma(L+B)$ denotes the infection force when the worm is entering a latent population, $\sigma(L+B) S$ is the number of susceptible groups that are infected, and $\sigma(L+$ $B) S(1-\mu)$ denotes the number of infected groups with normal function rate.

(2) "By vaccination strategies, all mobile devices are installed antivirus software. Hence, their immunity capability to worm is improved. However, antivirus software cannot guarantee that all devices are protected from worm attacks, so the injection of antivirus software is partial efficiency. Namely, there are $\varepsilon L(\varepsilon=v(1-\mu))$. Latent devices move to the $R$-status, where $v(1-\mu)$ denotes vaccination effective rate for normal function group. When $v=0$, the vaccination strategy has no effect at all; when $v=1$, the vaccination strategy is perfectly effective.

(3) The remaining $(1-v)$ fraction of latent devices has no immunity to worm attacks and then has to go to the $B$-status or fall into dysfunctional status. In other words, $\beta L(\beta=(1-v)(1-\mu) \Phi)$ latent devices transition to the $B$-status, where $\Phi(1-v)(1-\mu)$ denotes the rate when the worm begins actively scanning the networks for new victim.

(4) Considering the quarantine strategy imposed in the $B$-status, the infectious devices can be quarantined 
into the $Q$-status with a transfer rate $\gamma(\gamma=\phi(1-\mu))$, where $\phi(1-\mu)$ denotes quarantined effective rate for normal function group. When $\phi=0$, the quarantine strategy is not considered, and no infectious device is quarantined.

(5) Considering system reinstall strategy, some mobile devices can be selected for system reinstallation, which makes $\xi Q(\xi=(1-\mu) \psi)$ devices move form $Q$-status to $R$-status, where $(1-\mu) \psi$ denotes recovery effective rate for normal function group.

(6) Considering the variation of the virus, antivirus software is only temporary immune function. After the temporary immunization period $\tau$, $\varepsilon L(t-\tau) e^{-\mu \tau}+\xi Q(t-\tau) e^{-\mu \tau}$ devices will lose immunization and move from $R$-status to $S$-status.

(7) Device dysfunction occurs in all statuses because of the natural death; its death rate in the total devices is denoted by $\mu$.

According to different status transition relationships, we can formulate the SLBQR model by the following differential equations:

$$
\left\{\begin{array}{l}
\frac{\mathrm{d} S(t)}{\mathrm{d} t}=-\alpha S(t)(L(t)+B(t))-\mu S(t)+\mu N+\varepsilon L(t-\tau) e^{-\mu \tau}+\xi Q(t-\tau) e^{-\mu \tau}, \\
\frac{\mathrm{d} L(t)}{\mathrm{d} t}=\alpha S(t)(L(t)+B(t))-\mu L(t)-\beta L(t)-\varepsilon L(t), \\
\frac{\mathrm{d} B(t)}{\mathrm{d} t}=\beta L(t)-\gamma B(t)-\mu B(t), \\
\frac{\mathrm{d} Q(t)}{\mathrm{d} t}=\gamma B(t)-\mu Q(t)-\xi Q(t), \\
\frac{\mathrm{d} R(t)}{\mathrm{d} t}=\varepsilon L(t)+\xi Q(t)-\mu R(t)-\varepsilon L(t-\tau) e^{-\mu \tau}-\xi Q(t-\tau) e^{-\mu \tau} .
\end{array}\right.
$$

The feasible region of the system (1) can be described as $\Omega=\{(S, L, B, Q, R) \mid S, L, B, Q, R \geq 0, S+L+B+Q+R=N\}$,

which is a positively invariant set. By calculating system (1), we can obtain easily that system (1) always has a worm-free equilibrium, $P_{0}(N, 0,0,0,0)$. According to the theory of the next generation operator in [26], we can calculate the basic reproduction number $R_{0}$ as follows.

Let $\mathrm{d} L(t) / \mathrm{d} t=\alpha S(t)(L(t)+B(t))-L(t)(\beta+\varepsilon+\mu)=0$; then, $L(t)=\alpha S_{0} B(t) /\left(\beta \mu \varepsilon-\alpha S_{0}\right)$, where $S_{0}=N$. Thus,

$$
A=D_{B}\left(\frac{\mathrm{d} B(t)}{\mathrm{d} t}\right)=\left.D_{B}\left(\frac{\alpha \beta S_{0} B(t)}{\mu+\beta+\varepsilon-\alpha S_{0}}-(\mu+\gamma) B(t)\right)\right|_{B(t)=0}=\frac{\alpha \beta S_{0}}{\mu+\beta+\varepsilon-\alpha S_{0}}-(\mu+\gamma)=M-D
$$

where $M, D \geq 0, \alpha<(\mu+\beta+\varepsilon) / N$. Subsequently, we can give specific expression of basic reproduction number $R_{0}$ :

$$
R_{0}=M D^{-1}=\frac{\alpha \beta S_{0}}{\beta+\mu+\varepsilon-\alpha S_{0}} \cdot \frac{1}{\gamma+\mu}=\frac{\alpha \beta N}{(\gamma+\mu)(\mu+\beta+\varepsilon-\alpha N)} .
$$

Further, we can give the expression for quarantined rate $\varphi:$

$$
\varphi=\frac{\sigma(1-v)(1-\mu) \oplus N}{R_{0}(\mu+(1-v)(1-\mu) \omega+v(1-\mu)-\sigma(1-\mu) N)}-\frac{\mu}{1-\mu} .
$$

\section{Stability Analysis for Equilibrium}

In this section, firstly, we calculate system (1) and give expression of the worm-free equilibrium and the endemic equilibrium of system (1). Secondly, we investigate the effects of different quarantined rates on worm propagation and give the stability theorem of worm-free equilibrium and the endemic equilibrium in different in quarantined rates. In the end, we give the effects of immune duration on worm transmission and optimal quarantined strategy. 


\subsection{Equilibriums}

Theorem 1. System (1) exists in the unique worm-free equilibrium:

$$
P_{0}\left(S_{0}, L_{0}, B_{0}, Q_{0}, R_{0}\right)=(N, 0,0,0,0),
$$

and the unique endemic equilibrium:

$$
P^{*}=\left(S^{*}, L^{*}, B^{*}, Q^{*}, R^{*}\right)
$$

$$
\begin{aligned}
S^{*} & =\frac{(\gamma+\mu)(\mu+\varepsilon+\beta)}{\alpha(\gamma+\mu+\beta)}, \\
Q^{*} & =\frac{\mu(\gamma+\mu)(\mu+\beta+\varepsilon)-\mu \alpha N(\gamma+\mu+\beta)}{\left.\alpha(\gamma+\mu+\beta) e^{-\mu \tau}[\xi+(\varepsilon / \gamma \beta)(\gamma+\mu)(\mu+\xi)]-(\gamma+\mu)(\mu+\beta+\varepsilon)(\mu+\xi)((\gamma+\mu) / \beta \gamma)+(1 / \gamma)\right) \alpha}, \\
B^{*} & =\frac{1}{\gamma}(\mu+\xi) Q^{*}, \\
L^{*} & =\frac{1}{\beta \gamma}(\gamma+\mu)(\mu+\xi) Q^{*}, \\
R^{*} & =\left(1-e^{-\mu \tau}\right)\left[\frac{(\gamma+\mu)(\mu+\xi)}{\beta \gamma} \varepsilon-\xi\right] \frac{Q^{*}}{\mu} .
\end{aligned}
$$

Proof. Solving the following equation:

$$
\left\{\begin{array}{l}
\frac{\mathrm{d} S(t)}{\mathrm{d} t}=-\alpha S(t)(L(t)+B(t))-\mu S(t)+\mu N+\varepsilon L(t-\tau) e^{-\mu \tau}+\xi Q(t-\tau) e^{-\mu \tau}=0, \\
\frac{\mathrm{d} L(t)}{\mathrm{d} t}=\alpha S(t)(L(t)+B(t))-\mu L(t)-\beta L(t)-\varepsilon L(t)=0, \\
\frac{\mathrm{d} B(t)}{\mathrm{d} t}=\beta L(t)-\gamma B(t)-\mu B(t)=0, \\
\frac{\mathrm{d} Q(t)}{\mathrm{d} t}=\gamma B(t)-\mu Q(t)-\xi Q(t)=0, \\
\frac{\mathrm{d} R(t)}{\mathrm{d} t}=\varepsilon L(t)+\xi Q(t)-\mu R(t)-\varepsilon L(t-\tau) e^{-\mu \tau}-\xi Q(t-\tau) e^{-\mu \tau}=0 .
\end{array}\right.
$$

When $B(t)=0$, we can obtain the unique worm-free equilibrium $P_{0}\left(S_{0}, L_{0}, B_{0}, Q_{0}, R_{0}\right)=(N, 0,0,0,0)$; otherwise, we can obtain the unique endemic equilibrium $P^{*}=\left(S^{*}, L^{*}, B^{*}, Q^{*}, R^{*}\right)$. The proof is completed. 


$$
\begin{aligned}
S^{*} & =\frac{(\gamma+\mu)(\mu+\varepsilon+\beta)}{\alpha(\gamma+\mu+\beta)}, \\
Q^{*} & =\frac{\mu(\gamma+\mu)(\mu+\beta+\varepsilon)-\mu \alpha N(\gamma+\mu+\beta)}{\alpha(\gamma+\mu+\beta) e^{-\mu \tau}[\xi+(\varepsilon / \gamma \beta)(\gamma+\mu)(\mu+\xi)]-(\gamma+\mu)(\mu+\beta+\varepsilon)(\mu+\xi)((\gamma+\mu) / \beta \gamma n+q 1 / \gamma) \alpha}, \\
B^{*} & =\frac{1}{\gamma}(\mu+\xi) Q^{*}, \\
L^{*} & =\frac{1}{\beta \gamma}(\gamma+\mu)(\mu+\xi) Q^{*}, \\
R^{*} & =\left(1-e^{-\mu \tau}\right)\left[\frac{(\gamma+\mu)(\mu+\xi)}{\beta \gamma} \varepsilon-\xi\right] \frac{Q^{*}}{\mu} .
\end{aligned}
$$

\subsection{Stability of the Worm-Free Equilibrium}

Theorem 2. If $\varphi>((\sigma(1-v)(1-\mu) @ N) /(\mu+(1-v)(1-$ $\mu) \omega+v(1-\mu)-\sigma(1-\mu) N))-(\mu /(1-\mu)), P_{0}$ is locally asymptotically stable with respect to $\Omega$; otherwise, it is unstable.
Proof. According to Theorem 1, system (1) has a unique worm-free equilibrium $P_{0}=(N, 0,0,0,0)$. The Jacobian matrix at the worm-free equilibrium $P_{0}$ is

$$
J\left(P_{0}\right)=\left[\begin{array}{ccccc}
-\alpha\left(L_{0}+B_{0}\right)-\mu & -\alpha S_{0}+\varepsilon e^{-\mu \tau} & -\alpha S_{0} & \xi e^{-\mu \tau} & 0 \\
\alpha\left(L_{0}+B_{0}\right) & \alpha S_{0}-(\mu+\beta+\varepsilon) & \alpha S_{0} & 0 & 0 \\
0 & \beta & -(\mu+\gamma) & 0 & 0 \\
0 & 0 & \gamma & -(\mu+\xi) & 0 \\
0 & \varepsilon-\varepsilon e^{-\mu \tau} & 0 & \xi-\xi e^{-\mu \tau} & -\mu
\end{array}\right] .
$$

We can obtain the characteristic equation of $J\left(P_{0}\right)$ :

That is,

$$
\left|\lambda I-J\left(P_{0}\right)\right|=\left[\begin{array}{ccccc}
\lambda+\alpha\left(L_{0}+B_{0}\right)+\mu & \alpha S_{0}-\varepsilon e^{-\mu \tau} & \alpha S_{0} & -\xi e^{-\mu \tau} & 0 \\
-\alpha\left(L_{0}+B_{0}\right) & \lambda-\alpha S_{0}+(\mu+\beta+\varepsilon) & -\alpha S_{0} & 0 & 0 \\
0 & -\beta & \lambda+(\mu+\gamma) & 0 & 0 \\
0 & 0 & -\gamma & \lambda+(\mu+\xi) & 0 \\
0 & -\varepsilon+\varepsilon e^{-\mu \tau} & 0 & -\xi+\xi e^{-\mu \tau} & \lambda+\mu
\end{array}\right]=0 .
$$

$$
(\lambda+\mu)^{2}(\lambda+\mu+\xi)[(\lambda-\alpha N+\mu+\beta+\varepsilon)(\lambda+\gamma+\mu)-\alpha \beta N]=0 .
$$

where $a=\gamma+\mu, b=\mu+\beta+\varepsilon-\alpha N$.

Then, we can obtain

So, we calculate the eigenvalues of $J\left(P_{0}\right)$ : $\lambda_{1}=\lambda_{2}=-\mu, \lambda_{3}=-\mu-\xi, \lambda_{4}, \lambda_{5}$, where $\lambda_{4}, \lambda_{5}$ are the roots of equation (14) as follows:

$$
\begin{aligned}
& H_{1}=a+b=\gamma+\mu+\mu+\beta+\varepsilon-\alpha N>0 \\
& H_{2}=(a+b)(a b-\alpha \beta N)=(a+b)[(\gamma+\mu)(\mu+\beta+\varepsilon-\alpha N)-\alpha \beta N] .
\end{aligned}
$$


Because $\quad \varphi>((\sigma(1-v)(1-\mu) \oplus N) /(\mu+(1-v)(1-$ $\mu) \Phi+v(1-\mu)-\sigma(1-\mu) N))-(\mu /(1-\mu))$, we can obtain $\gamma>(\alpha \beta N /(\mu+\beta+\varepsilon-\alpha N))-\mu$; hence, $H_{2}>0$.

By referring to the Hurwitz criterion [27], we know that all roots of equation (14) have negative real parts. That is, all real parts of roots of equation (13) are negative. Hence, by referring to stability theory [27], if $\varphi>((\sigma(1-$ $v)(1-\mu) @ N) /(\mu+(1-v)(1-\mu) \quad \varpi+v(1-\mu)-\sigma(1-\mu)$ $N))-(\mu /(1-\mu))$, we can know $P_{0}$ is locally asymptotically stable. Otherwise, $P_{0}$ is unstable. Thus, the theory is proved.
Theorem 3. If $\varphi>((\sigma(1-v)(1-\mu) \bowtie N) /(\mu+(1-v)(1-$ $\mu) \emptyset+v(1-\mu)-\sigma(1-\mu) N))-(\mu /(1-\mu)), P_{0}$ is globally asymptotically stable with respect to $\Omega$; otherwise, $P_{0}$ is unstable.

Proof. Firstly, we construct a Lyapunov function:

$$
V(t)=\beta L(t)+(\mu+\beta+\varepsilon-\alpha N) B(t) .
$$

Then,

$$
\begin{aligned}
\frac{\mathrm{d} V(t)}{\mathrm{d} t} & =\beta \frac{\mathrm{d} L(t)}{\mathrm{d} t}+(\mu+\beta+\varepsilon-\alpha N) \frac{\mathrm{d} B(t)}{\mathrm{d} t} \\
& =\beta[\alpha S(t)(L(t)+B(t))-(\mu+\beta+\varepsilon) L(t)]+(\mu+\beta+\varepsilon-\alpha N)[\beta L(t)-(\gamma+\mu) B(t)] \\
& =[\alpha \beta S(t)-(\mu+\beta+\varepsilon-\alpha N)(\gamma+\mu)] B(t)+\beta L(t)[\alpha S(t)-(\mu+\beta+\varepsilon)+(\mu+\beta+\varepsilon-\alpha N)] \\
& \leq[\alpha \beta N-(\mu+\beta+\varepsilon-\alpha N)(\gamma+\mu)] B(t) .
\end{aligned}
$$

Because

$$
\varphi>\frac{\sigma(1-v)(1-\mu) \bowtie N}{(\mu+(1-v)(1-\mu) \Phi+v(1-\mu)-\sigma(1-\mu) N)}-\frac{\mu}{1-\mu},
$$

we can obtain

$$
(\gamma+\mu)(\mu+\beta+\varepsilon-\alpha N)>\alpha \beta N
$$

Hence, $\mathrm{d} V(t) / \mathrm{d} t<0$ with $\Omega$. By referring to LaSalle invariance principle [28], we can prove Theorem 3 .

3.3. Stability of the Endemic Equilibrium. Now, we will analyze the stability of the endemic equilibrium: $P^{*}=\left(S^{*}, L^{*}, B^{*}, Q^{*}, R^{*}\right)$, where

$$
\begin{aligned}
S^{*} & =\frac{(\gamma+\mu)(\mu+\varepsilon+\beta)}{\alpha(\gamma+\mu+\beta)}, \\
Q^{*} & =\frac{\mu(\gamma+\mu)(\mu+\beta+\varepsilon)-\mu \alpha N(\gamma+\mu+\beta)}{\alpha(\gamma+\mu+\beta) e^{-\mu \tau}[\xi+(\varepsilon / \gamma \beta)(\gamma+\mu)(\mu+\xi)]-(\gamma+\mu)(\mu+\beta+\varepsilon)(\mu+\xi)(((\gamma+\mu) / \beta \gamma)+(1 / \gamma)) \alpha}, \\
B^{*} & =\frac{1}{\gamma}(\mu+\xi) Q^{*} \\
L^{*} & =\frac{1}{\beta \gamma}(\gamma+\mu)(\mu+\xi) Q^{*}, \\
R^{*} & =\left(1-e^{-\mu \tau}\right)\left[\frac{(\gamma+\mu)(\mu+\xi)}{\beta \gamma} \varepsilon-\xi\right] \frac{Q^{*}}{\mu} .
\end{aligned}
$$

Obviously, $Q^{*}>0, B^{*}>0, R^{*}>0, L^{*}>0$; thus $N>S^{*}$, so we can obtain $N / S^{*}=(\alpha \beta N /((\gamma+\mu)(\mu+\beta+\varepsilon)))+$ $(\alpha N /(\mu+\beta+\varepsilon))>1$. Then, multiply both sides of this inequality by $(\mu+\beta+\varepsilon) /(\mu+\beta+\varepsilon-\alpha N)$, and we can obtain $\gamma<(\alpha \beta N /(\mu+\beta+\varepsilon-\alpha N))-\mu$. Further, we can obtain $\varphi<((\sigma(1-v)(1-\mu) @ N) /(\mu+(1-v)(1-\mu) \emptyset+v(1-\mu)-$ $\sigma(1-\mu) N))-(\mu /(1-\mu))$.
Theorem 4. $P^{*}$ is locally asymptotically stable with respect to $\Omega$ if $\varphi<((\sigma(1-v)(1-\mu) \bowtie N) /(\mu+(1-v)(1-\mu) \emptyset+v(1-$ $\mu)-\sigma(1-\mu) N))-(\mu /(1-\mu)), \tau \geq 0$.

Proof. Because $P^{*}=\left(S^{*}, L^{*}, B^{*}, Q^{*}, R^{*}\right)$, we can obtain the Jacobian matrix at $P^{*}$ as follows: 


$$
J\left(P^{*}\right)=\left[\begin{array}{ccccc}
-\alpha\left(L^{*}+B^{*}\right)-\mu & -\alpha S^{*}+\varepsilon e^{-\mu \tau} & -\alpha S^{*} & \xi e^{-\mu \tau} & 0 \\
\alpha\left(L^{*}+B^{*}\right) & \alpha S^{*}-(\mu+\beta+\varepsilon) & \alpha S^{*} & 0 & 0 \\
0 & \beta & -(\mu+\gamma) & 0 & 0 \\
0 & 0 & \gamma & -(\mu+\xi) & 0 \\
0 & \varepsilon-\varepsilon e^{-\mu \tau} & 0 & \xi-\xi e^{-\mu \tau} & -\mu
\end{array}\right] .
$$

We can obtain the characteristic equation of $J\left(P^{*}\right)$ :

$$
\left|\lambda I-J\left(P^{*}\right)\right|=\left[\begin{array}{ccccc}
\lambda+\alpha\left(L^{*}+B^{*}\right)+\mu & \alpha S^{*}-\varepsilon e^{-\mu \tau} & \alpha S^{*} & -\xi e^{-\mu \tau} & 0 \\
-\alpha\left(L^{*}+B^{*}\right) & \lambda-\alpha S^{*}+(\mu+\beta+\varepsilon) & -\alpha S^{*} & 0 & 0 \\
0 & -\beta & \lambda+(\mu+\gamma) & 0 & 0 \\
0 & 0 & -\gamma & \lambda+(\mu+\xi) & 0 \\
0 & -\varepsilon+\varepsilon e^{-\mu \tau} & 0 & -\xi+\xi e^{-\mu \tau} & \lambda+\mu
\end{array}\right]
$$

That is,

$$
\begin{aligned}
& (\lambda+\mu)\left(\lambda+\alpha\left(L^{*}+B^{*}\right)+\mu\right)(\lambda+\mu+\xi)\left[[ ( \lambda - \alpha S ^ { * } + \mu + \beta + \varepsilon ) ( \lambda + \gamma + \mu ) - \alpha \beta S ^ { * } ] \alpha ( L ^ { * } + B ^ { * } ) \left[\left(\alpha S^{*}-\varepsilon e^{-\mu \tau}\right)(\lambda+\gamma+\mu)(\lambda\right.\right. \\
& \left.\left.\quad+\mu+\xi)+\beta\left[\alpha S^{*}(\lambda+\mu+\xi)-\gamma \xi e^{-\mu \tau}\right]\right]\right]=0 .
\end{aligned}
$$

$$
\lambda^{4}+a \lambda^{3}+b \lambda^{2}+c \lambda+d=0
$$

So, we can calculate the eigenvalues of $J\left(P^{*}\right)$ : where $\lambda_{1}=-\mu, \lambda_{2}, \lambda_{3}, \lambda_{4}, \lambda_{5}$, where $\lambda_{2}, \lambda_{3}, \lambda_{4}, \lambda_{5}$ are the roots of equation (24) as follows:

$$
\begin{aligned}
a= & \frac{\alpha\left(L^{*}+B^{*}\right)+4 \mu+\xi+\beta+\varepsilon-\alpha S^{*}+\gamma}{\alpha}, \\
b= & \left(\alpha\left(L^{*}+B^{*}\right)+\mu\right)\left(3 \mu+\xi+\beta+\varepsilon-\alpha S^{*}+\gamma\right)+(\mu+\xi)\left(\mu+\beta+\varepsilon-\alpha S^{*}\right)+\alpha\left(L^{*}+B^{*}\right)\left(\alpha S^{*}-\varepsilon e^{-\mu \tau}\right) \\
& +(\mu+\xi)(\gamma+\mu)+\left(\mu+\beta+\varepsilon-\alpha S^{*}\right)(\gamma+\mu)-\alpha \beta S^{*}, \\
c= & \alpha^{2} \beta\left(L^{*}+B^{*}\right) S^{*}+\alpha\left(L^{*}+B^{*}\right)\left(\alpha S^{*}-\varepsilon e^{-\mu \tau}\right)(\gamma+2 \mu+\xi)-\alpha \beta S^{*}(\mu+\xi)-\alpha \beta S^{*}\left(\alpha\left(L^{*}+B^{*}\right)+\mu\right), \\
d= & \left(\alpha\left(L^{*}+B^{*}\right)+\mu\right)\left(\mu+\beta+\varepsilon-\alpha S^{*}\right)(\gamma+\mu)+\alpha\left(L^{*}+B^{*}\right)\left(\alpha S^{*}-\varepsilon e^{-\mu \tau}\right)(\gamma+\mu)(\mu+\xi) \\
& +\alpha^{2} \beta S^{*}\left(L^{*}+B^{*}\right)(\mu+\xi)-\alpha \beta \gamma \xi\left(L^{*}+B^{*}\right) e^{-\mu \tau}-\alpha \beta S^{*}\left(\alpha\left(L^{*}+B^{*}\right)+\mu\right)(\mu+\xi) .
\end{aligned}
$$

For any $\tau \geq 0$, when $\varphi<((\sigma(1-v)(1-\mu) @ N) /(\mu+(1-$ $v)(1-\mu) \emptyset+v(1-\mu)-\sigma(1-\mu) N))-(\mu /(1-\mu))$, we can obtain $\gamma<(\alpha \beta N /(\mu+\beta+\varepsilon-\alpha N))-\mu$. Further, we can obtain

$$
\begin{aligned}
& H_{1}=a>0, \\
& H_{2}=a b-c>0, \\
& H_{3}=a(b c-a d)-c^{2}>0 .
\end{aligned}
$$

By referring to the Hurwitz criterion, we can know all real parts of roots of equation (24) are negative. That is, all real parts of roots of equation (23) are negative. Hence, by referring to stability theory, if $\varphi<((\sigma(1-v)(1-\mu)$ $\varpi N) /(\mu+(1-v)(1-\mu) \emptyset+v(1-\mu)-\sigma \quad(1-\mu) N))-$ $(\mu /(1-\mu))$, we can know $P^{*}$ is locally asymptotically stable. Otherwise, $P^{*}$ is unstable. Thus, the theory is proved. 


\subsection{Immune Time and Quarantined Strategy}

Theorem 5. The dynamic system (1) can just obtain temporary immunity time. The longer the immunity time $\tau$ is, the smaller the impact on the value of the endemic equilibrium is.

Proof. Obviously, according to equation (13), the time delay $\tau$ has no effect on the stability at $\mathrm{P}_{0}$. According to equation (24), when $\tau \geq 0$, whether $H_{1}, H_{2}$, and $H_{3}$ are greater than 0 is not affected by the value. Therefore, the value of $\tau$ will not affect the stability of $P^{*}$. Further, through the expression of $S^{*}, L^{*}, B^{*}, Q^{*}$, and $R^{*}$, we find that the value of $\tau$ does not affect the value of $S^{*}$. With the increase of $\tau$, the smaller the change of $e^{-\mu \tau}$ is, the smaller the impact on $L^{*}, B^{*}, Q^{*}$, and $R^{*}$ is. Thus, the theory is proved.

Theorem 6. $\varphi^{*}=(\sigma(1-v)(1-\mu) \bowtie N /(\mu+(1-v)(1-\mu)$ $\Phi+v(1-\mu)-\sigma(1-\mu) N))-(\mu /(1-\mu))$ is the threshold of quarantined rate, and when $\varphi=\varphi^{*}$,
$P_{1}=P_{0}=P^{*}=(N, 0,0,0,0)$ is globally asymptotically stable with respect to $\Omega$.

Proof. By Theorems 2-4, we know that $\varphi^{*}=(\sigma(1-v)(1-$ $\mu) \bowtie N /(\mu+(1-v)(1-\mu) \omega+v(1-\mu)-\sigma(1-\mu) N))-(\mu /$ $(1-\mu))$ is the boundary between the stability of the two fixed points, and thus $\varphi^{*}=(\sigma(1-v)(1-\mu) \bowtie N /(\mu+(1-$ $v)(1-\mu) \omega+v(1-\mu)-\sigma(1-\mu) N))-(\mu /(1-\mu))$ is the threshold of quarantined rate. Next, we prove the stability of $P_{1}$. Because $\varphi=(\sigma(1-v)(1-\mu) \omega N /(\mu+(1-v)(1-\mu) \omega+$ $v(1-\mu)-\sigma(1-\mu) N))-(\mu /(1-\mu))$, we can obtain $\gamma=(\alpha \beta N /(\mu+\beta+\varepsilon-\alpha N))-\mu$.

When $\gamma=(\alpha \beta N /(\mu+\beta+\varepsilon-\alpha N))-\mu$, we can obtain $P_{1}=P_{0}=P^{*}=(N, 0,0,0,0)$. That is, equation (1) only has one fixed point. It is worth noting that the stability of equation (1) at the above fixed point cannot be judged by Theorems 3 and 4. So, next we linearize equation (1) at $P_{1}$ and obtain the following characteristic matrix:

$$
A=\left[\begin{array}{ccccc}
-\mu & -\alpha N & \varepsilon e^{-\mu \tau} & \xi e^{-\mu \tau} & 0 \\
0 & \alpha N-\mu-\beta-\varepsilon & \alpha N & 0 & 0 \\
0 & \beta & -\frac{\alpha \beta N}{\mu+\beta+\varepsilon-\alpha N} & 0 & 0 \\
0 & 0 & \frac{\alpha \beta N}{\mu+\beta+\varepsilon-\alpha N}-\mu & -\xi-\mu & 0 \\
0 & \varepsilon-\varepsilon e^{-\mu \tau} & 0 & \xi-\xi e^{-\mu \tau} & 0
\end{array}\right] .
$$

According to $|\lambda E-A|=0$, we can obtain characteristic equation:

$$
\lambda(\lambda+\mu)(\lambda+\xi+\mu)\left[\left(\lambda+\frac{\alpha \beta N}{\mu+\beta+\varepsilon-\alpha N}\right)(\lambda-\alpha N+\mu+\beta+\varepsilon)-\alpha \beta N\right]=0
$$

Further, we can obtain characteristic roots:

$$
\begin{aligned}
& \lambda_{1}=\lambda_{2}=0, \\
& \lambda_{3}=-(\xi+\mu), \\
& \lambda_{4}=-\frac{\alpha N \beta+(\mu+\beta+\varepsilon-\alpha N)^{2}}{\mu+\beta+\varepsilon-\alpha N} .
\end{aligned}
$$

However, because there are zero characteristic roots, we cannot judge the stability of the equations in $P_{1}$. Therefore, we can only give the stability of equation (1) in $P_{1}$ by qualitative analysis. First, we choose an infinitesimal constant $\Delta$, satisfying

$$
\varphi=\varphi^{*}+\Delta \longrightarrow \frac{\sigma(1-v)(1-\mu) \bowtie N}{(\mu+(1-v)(1-\mu) \omega+v(1-\mu)-\sigma(1-\mu) N)}-\frac{\mu}{1-\mu}(\Delta \longrightarrow 0)
$$


Then, let $\Delta \longrightarrow 0^{+}$; we can obtain $\varphi=\varphi^{*}+\Delta>((\sigma(1-$ $v)(1-\mu) \omega N) /(\mu+(1-v)(1-\mu) \omega+v(1-\mu)-\sigma(1-\mu) N))-$ $(\mu /(1-\mu))$. According to Theorem $3, P_{1}$ is globally asymptotically stable. Finally, let $\Delta \longrightarrow 0^{-}$, and we can obtain $\varphi=\varphi^{*}+\Delta<((\sigma(1-v)(1-\mu) \bowtie N) /(\mu+(1-v)(1-\mu)$ $\omega+v(1-\mu)-\sigma(1-\mu) N))-(\mu /(1-\mu))$. According to Theorem $4, P_{1}$ is local asymptotically stable. So, when $\varphi=\varphi^{*}=$ $((\sigma(1-v)(1-\mu) \omega N) /(\mu+(1-v)(1-\mu) \omega+v(1-\mu)-\sigma(1-$ $\mu) N))-(\mu /(1-\mu)), P_{1}$ is globally asymptotically stable.

Theorem 7. Under mobile Internet, the base station should keep the quarantined rate within the range $\phi \geq \phi^{*}$. Within this range, the evolution of nonlinear dynamical systems (1) meets the company's ideal requirements.

Proof. See Theorems 2, 3, and 6 for proof.

\section{Simulation}

We use the first experiment to verify Theorem 2 and 3 . We suppose that the number of susceptible, latent, breaking-out, quarantined, and recovered nodes is

$$
\begin{aligned}
& S(0)=40000, \\
& L(0)=30000, \\
& B(0)=30000, \\
& Q(0)=0, \\
& R(0)=0 .
\end{aligned}
$$

And the parameters of system (1) are references [19]:

$$
\begin{aligned}
\sigma & =5.26 \times 10^{-7}, \\
v & =3.16 \times 10^{-7}, \\
\oplus & =3.16 \times 10^{-7}, \\
\varphi & =5.2 \times 10^{-2}, \\
\psi & =5.2 \times 10^{-2}, \\
\alpha & =5 \times 10^{-7}, \\
\beta & =3 \times 10^{-7}, \\
\varepsilon & =3 \times 10^{-7}, \\
\gamma & =5 \times 10^{-2}, \\
\xi & =5 \times 10^{-2}, \\
\mu & =5 \times 10^{-2}, \\
N & =10^{5}, \\
\tau & =10^{2} .
\end{aligned}
$$

According to the threshold expression of the quarantined rate, we can calculate

$$
\varphi^{*}=\frac{\sigma(1-v)(1-\mu) \omega N}{(\mu+(1-v)(1-\mu) \omega+v(1-\mu)-\sigma(1-\mu) N)}-\frac{\mu}{(1-\mu)}=-2.6 \times 10^{-2}
$$

Obviously, $\varphi=5.2 \times 10^{-2}>\varphi^{*}=-2.6 \times 10^{-2}$. From Figure 4 , we can see that worm propagation eventually stabilizes in the status $P_{0}$. That is, if $\varphi>\varphi^{*}$, status $P_{0}$ is stable, which verifies Theorems 2 and 3 .

Compared with Jia and Jing [16], the worm-free equilibrium in [16] will stabilize to susceptible and recovered statuses. First of all, their simulation results contradicted their conclusions. In their research, they pointed out that the system should be stable in a susceptible status; however, the final simulation results are stable in the susceptible status and recovery status. Second, this conclusion is unrealistic because the virus is constantly mutating, so the device can only obtain temporary immunity. Finally, even if their assumption is reasonable, that is, the device can obtain permanent immunity, then the system evolution should be stable in the recovery status rather than two statuses. Different from them, our simulation results show that in the case of temporary immune mechanism, the recovery status is only a transition status because with the continuous mutation of the virus, the recovery status will gradually become susceptible, which is consistent with the actual situation.

In the second experiment, we want to verify Theorems 4 and 5. Similar to the first experiment, we suppose that the number of susceptible, latent, breaking-out, quarantined, and recovered nodes is

$$
\begin{aligned}
& S(0)=40000, \\
& L(0)=30000, \\
& B(0)=30000, \\
& Q(0)=0, \\
& R(0)=0 .
\end{aligned}
$$

And the parameters of system (1) are 


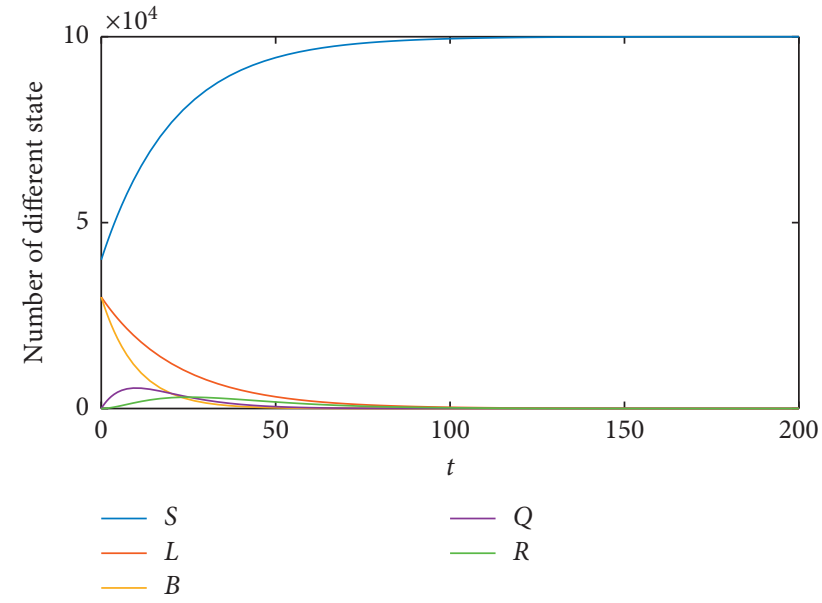

FIGURE 4: Stability of the worm-free equilibrium.

$$
\begin{aligned}
\sigma & =8.42 \times 10^{-7}, \\
v & =3.16 \times 10^{-4}, \\
\omega & =3.16 \times 10^{-2}, \\
\varphi & =5.26 \times 10^{-2}, \\
\psi & =5.26 \times 10^{-2}, \\
\alpha & =8 \times 10^{-7}, \\
\beta & =3 \times 10^{-2}, \\
\varepsilon & =3 \times 10^{-4}, \\
\gamma & =5 \times 10^{-2}, \\
\xi & =5 \times 10^{-2}, \\
\mu & =5 \times 10^{-2}, \\
N & =10^{5}, \\
\tau & =0 \\
\tau & =10 \\
\tau & =100 \\
\tau & =1000
\end{aligned}
$$

Now, we can calculate $\varphi^{*}=0.789$; obviously, $\varphi=5.26 \times 10^{-2}<\varphi^{*}$. From Figure 5, we can see that worm propagation eventually stabilizes in the status $P^{*}$. That is, if $\varphi<\varphi^{*}$, with the development of time, the system is always stable to $P^{*}$, which verifies Theorem 4 .

Then, by comparing Figures 5 and 6, we can see that mobile devices with temporary immunity will affect the number of different statuses, for example, the number of mobile devices in the infection status (latent status and breaking-out status) decreased. That is, vaccination strategies with temporary immunity can protect some devices from worm attacks. Next, by comparing Figures 6-8, we can see that when the immune time increases to a certain extent, it will not affect the status of the infection and the immune status. This is the reality

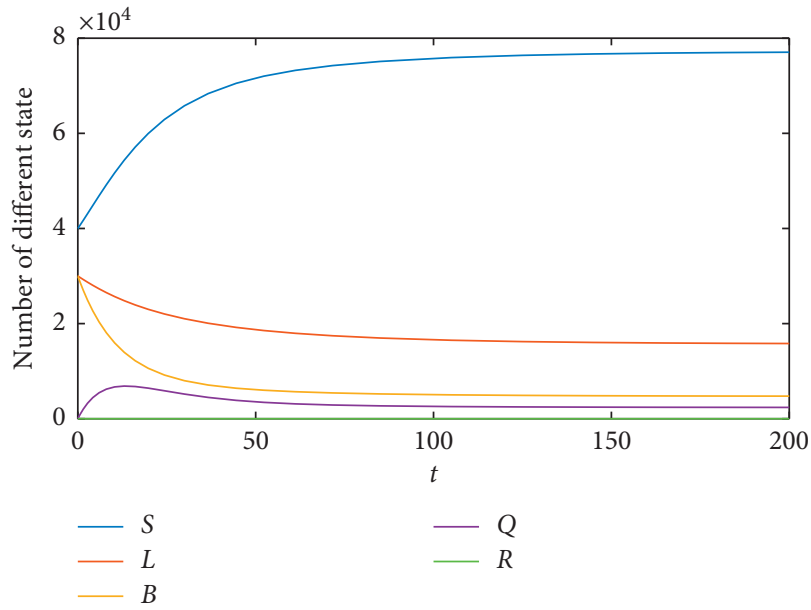

Figure 5: Stability of the endemic equilibrium $(\tau=0)$.

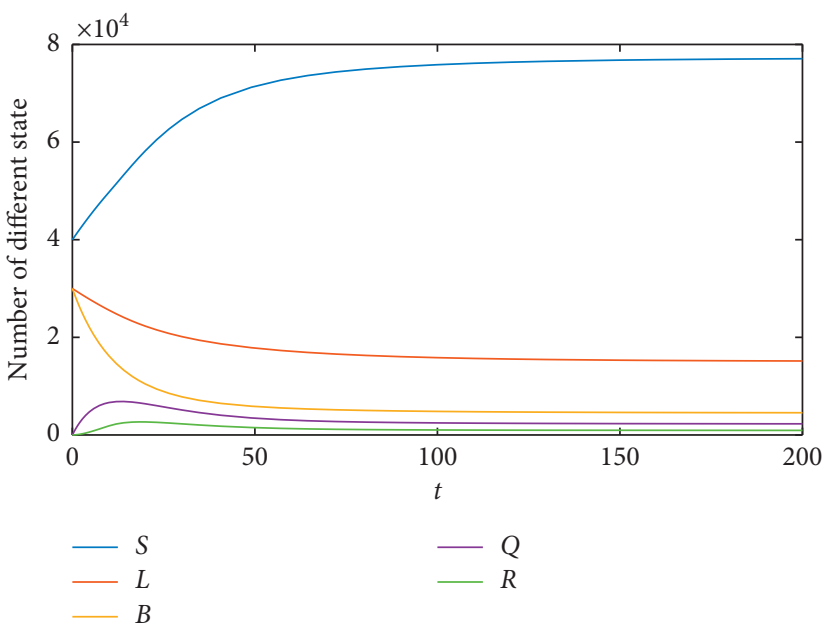

FIgURE 6: Stability of the endemic equilibrium $(\tau=10)$.

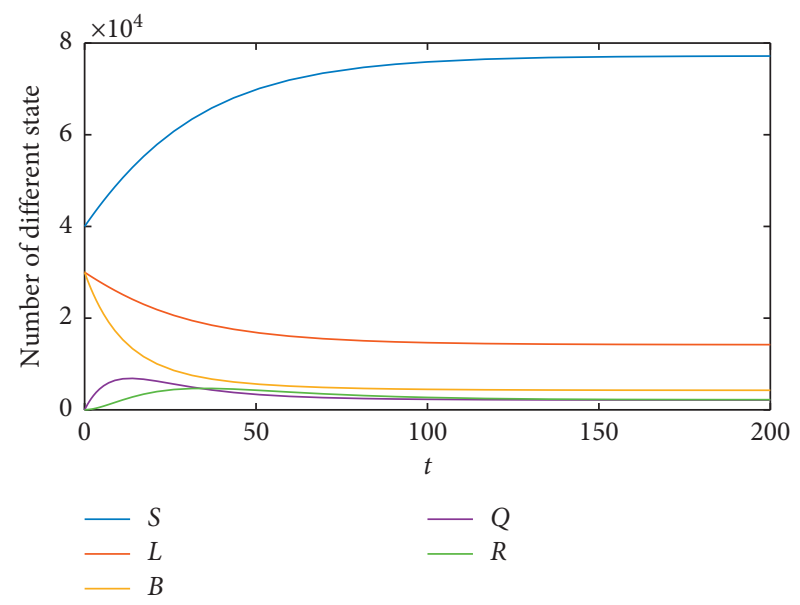

FIgURE 7: Stability of the endemic equilibrium $(\tau=100)$.

because when the immune time is very long, the virus will mutate, so the immune effect of the mobile device will fail, which verifies Theorem 5 . 


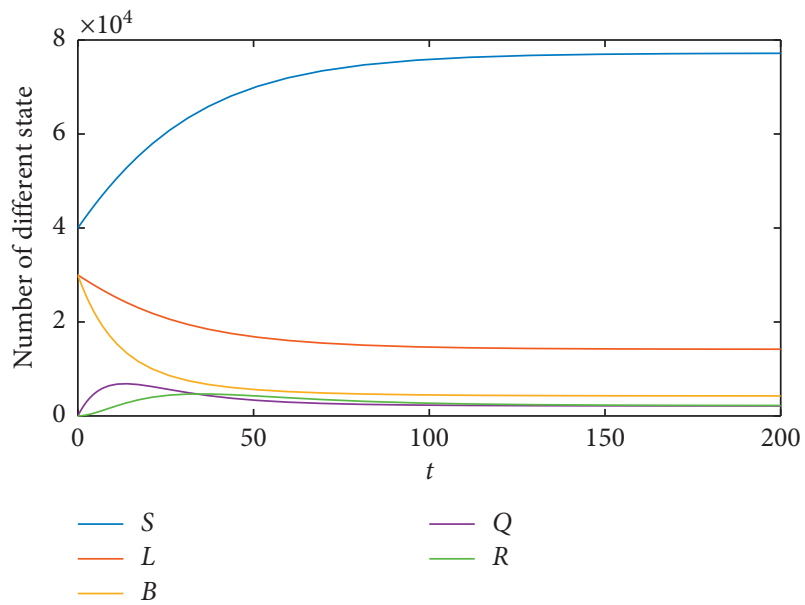

Figure 8: Stability of the endemic equilibrium $(\tau=1000)$.

Compared with Jia and Jing [16], the figure of stability of the endemic equilibrium is wrong in [16] because in the figure of stability of the endemic equilibrium, they point out that the system eventually maintains a susceptible status, which means $P_{0}=P^{*}$. Obviously, this is contrary to their conclusions. Our results not only validate the stability of the endemic equilibrium but also point out the variability of the virus because we can see that the longer the temporary immunization time, the smaller the impact on the evolution of the virus, which means that the virus will mutate.

In the last experiment, in order to research the influence of the different configuring quarantine policy to worm propagation considering time delay, different quarantined rates are used to observe the number of infected nodes in the evolution process of system (1). Similar to the above two experiments, we suppose that the number of susceptible, latent, breaking-out, quarantined, and recovered nodes is

$$
\begin{aligned}
S(0) & =60000, \\
L(0) & =40000, \\
B(0) & =0, \\
Q(0) & =0, \\
R(0) & =0 .
\end{aligned}
$$

And the parameters of system (1) are

$$
\begin{aligned}
\alpha & =0.0000008, \\
\beta & =0.03 \\
\varepsilon & =0.003 \\
\xi & =0.05 \\
\mu & =0.05 \\
N & =100000 \\
\tau & =100 .
\end{aligned}
$$

In this experiment, there were three situations:
Situation 1: $\varphi=\varphi^{*}=((\sigma(1-v)(1-\mu) \emptyset N) /(\mu+(1-$ $v)(1-\mu) @+v(1-\mu)-\sigma(1-\mu) N))$ $-(\mu /(1-\mu))=0.79$.

Situation 2: $\phi=0.74<\phi^{*}$.

Situation 3: $\phi=0.84>\phi^{*}$.

The result is shown in Figure 9. Through the second subgraph of Figure 9, we can see that when our quarantined rate is less than the threshold $\phi^{*}$, the worm cannot be completely removed, and the infected mobile device amount eventually stabilizes at about 750 . Through the first subgraph, the third subgraph, and the fourth subgraph, we can see that when our quarantined rate is not less than the threshold $\phi^{*}$, the worm can be completely removed. This result also verifies Theorems 6 and 7 .

Compared with Jia and Jing [16], we give specific quarantined strategies and quarantined thresholds and verify that when the quarantined value of the system is near the threshold, it will not cause the system to fork or chaos.

\section{Discussion}

In this paper, we mainly solve the following problems:

(1) We prove that the vaccination strategy is impossible to have full efficacy and permanent immunity.

(2) We give the expression of the quarantined rate of expression, discuss the impact of different quarantined strategies on the spread of worms, and give what kind of quarantined strategy is to be adopted to completely suppress the spread of the virus.

(3) Existing literature ignores $R_{0}=1$ when they calculate the basic reproduction number $R_{0}$; we discuss the situation $R_{0}=1$.

In solving the above problems, the research methods we adopted are as follows:

(1) Based on basic reproduction number, we give expression of quarantined rate $\varphi$.

(2) By deriving the local and global stabilities in both the worm-free and endemic equilibrium, we prove the worm-free equilibrium is locally and globally asymptotically stable if $\phi \geq((\sigma(1-v)(1-$ $\mu) \bowtie N) /\left(R_{0}(\mu+(1-v)(1-\mu) \varpi+v(1-\mu)-\sigma(1-\right.$ $\mu) N)))-(\mu /(1-\mu))$, whereas the endemic equilibrium is locally asymptotically stable if $\phi<((\sigma)(1-$ $v)(1-\mu) \emptyset N) /\left(R_{0}(\mu+(1-v)(1-\mu) \Phi+v(1-\mu)-\right.$ $\sigma(1-\mu) N)))-(\mu /(1-\mu))$. The worm-free equilibrium is asymptotically stable, implying that the worm dies out eventually and its attack remains under control; the endemic equilibrium is asymptotically stable, implying that the worm is always persistent and spreading within a population. Hence, we can give threshold expression $\phi^{*}=((\sigma(1-v)$ $(1-\mu) \oplus N) /\left(R_{0}(\mu+(1-v) \quad(1-\mu) \Phi+v(1-\mu)-\right.$ $\sigma(1-\mu) N)))-(\mu /(1-\mu))$.

(3) First, we choose an infinitesimal constant $\Delta$, satisfying 

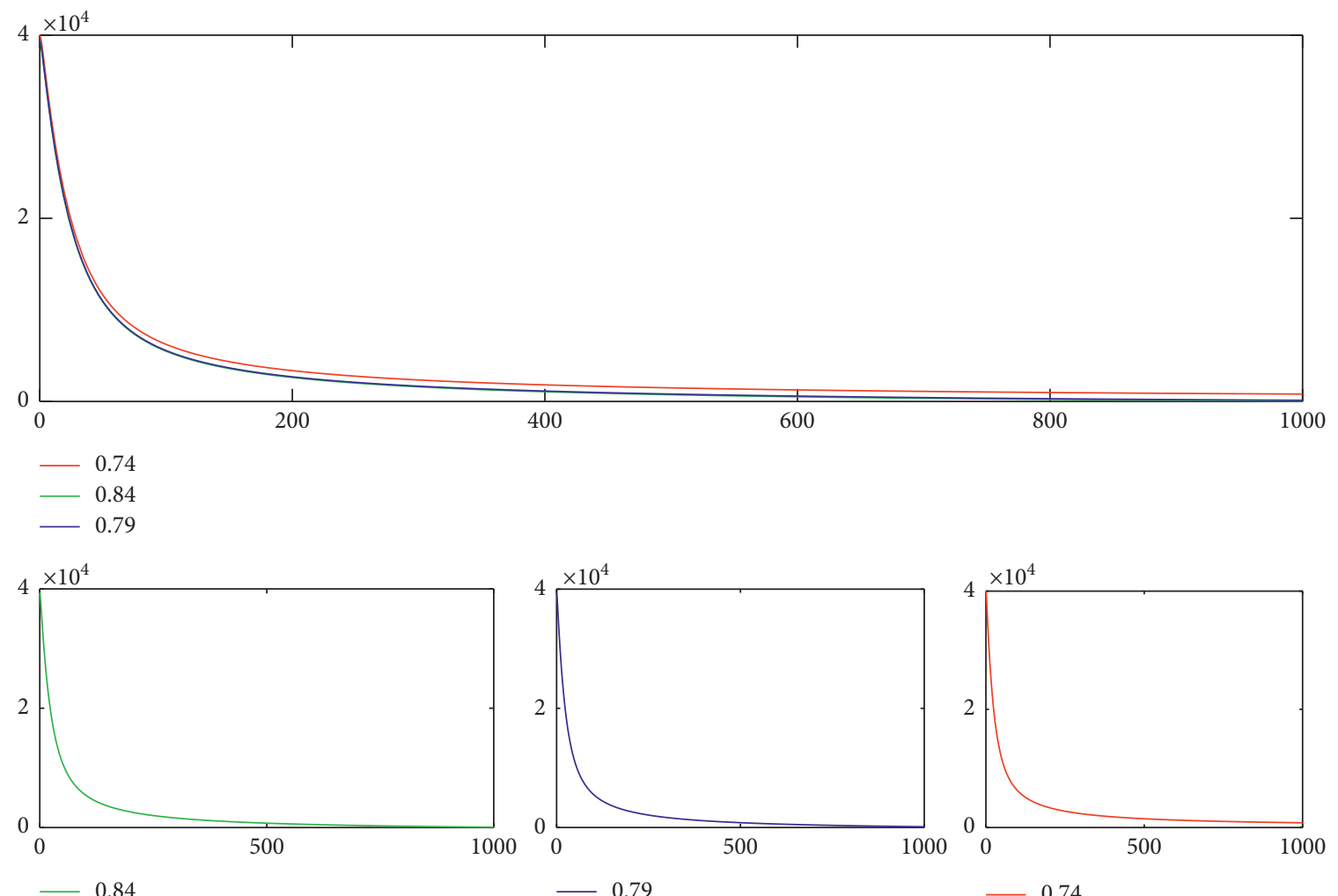

Figure 9: Impact of the quarantine rate on the infected nodes.

$$
\varphi=\varphi^{*}+\Delta \longrightarrow \frac{\sigma(1-v)(1-\mu) \bowtie N}{(\mu+(1-v)(1-\mu) Ф+v(1-\mu)-\sigma(1-\mu) N)}-\frac{\mu}{1-\mu}(\Delta \longrightarrow 0)
$$

Then, let $\Delta \longrightarrow 0^{+}$, and we can obtain $\varphi=\varphi^{*}+\Delta>((\sigma(1-v)(1-\mu) \omega N) /(\mu+(1-v)(1-\mu)$ $\omega+v(1-\mu)-\sigma(1-\mu) N))-(\mu /(1-\mu))$. According to Theorem $3, P_{1}$ is globally asymptotically stable. Finally, let $\Delta \longrightarrow 0^{-}$, and we can obtain $\varphi=\varphi^{*}+\Delta<((\sigma(1-v)(1-\mu) \Phi N) /(\mu+(1-v) \quad(1-$ $\mu) \omega+v(1-\mu)-\sigma(1-\mu) N))-(\mu /(1-\mu))$. According to Theorem $4, P_{1}$ is local asymptotically stable. So, when $\varphi=\varphi^{*}=((\sigma(1-v)(1-\mu) \Phi N) /(\mu+(1-v)$ $(1-\mu) @+v(1-\mu)-\sigma(1-\mu) N))-(\mu /(1-\mu)), P_{1}$ is globally asymptotically stable.

\section{Conclusion}

In this paper, we established the SLBQR model for worm propagation in mobile Internet considering time delay and quarantined rate. Our research has important academic value for subsequent scholars to study such issues. First of all, our research proves that there is no vaccination strategy for permanent immunity. Secondly, a quarantined strategy that suppresses the spread of viruses can help manufacturers to isolate equipment reasonably and reduce economic losses. Finally, our research provides an important research direction for the follow-up research: how to give the optimal strategy of quarantine under consideration of the minimum economic loss of the enterprise.

However, our research still has the following disadvantages:

(1) In this paper, we just introduce vaccination effective rate, and there is no discussion and analysis of the specific vaccination strategy. In this regard, we will conduct a specific analysis of the vaccination effective rate by introducing a vaccination rate in subsequent studies.

(2) We only give the threshold of immunity and simple quarantined strategy, without considering the impact of quarantined cost. Our future work will explore how to determine the appropriate quarantined rate with the least cost of quarantine.

\section{Data Availability}

The data used in this study are included in this article.

\section{Conflicts of Interest}

The authors declare that they have no conflicts of interest. 


\section{Acknowledgments}

This study was sponsored by the Guangdong Provincial National Science Foundation Project (2018A030313079) and 2018 Guangzhou Philosophy and Social Science Development "13th Five Year Plan" (2018GZYB16).

\section{References}

[1] A. Kumar Sangaiah, A. A. R. Hosseinabadi, M. B. Shareh, S. Y. B. Rad, A. Zolfagharian, and N. Chilamkurti, "IoT resource allocation and optimization based on heuristic algorithm," Sensors, vol. 20, no. 2, pp. 1-26, 2020.

[2] A. K. Sangaiah, M. Sadeghilalimi, A. A. R. Hosseinabadi, and W. Zhang, "Energy consumption in point-coverage wireless sensor networks via Bat algorithm," IEEE Access, vol. 7, pp. 180258-180269, 2019.

[3] J. Huang, "Ip flow mobility in the industry: from an economic perspective," IEEE Access, vol. 5, no. 99, pp. 53055-53068, 2017.

[4] A. K. Sangaiah, D. V. Medhane, G.-B. Bian, A. Ghoneim, M. Alrashoud, and M. S. Hossain, "Energy-aware green adversary model for cyberphysical security in industrial system," IEEE Transactions on Industrial Informatics, vol. 16, no. 5, pp. 3322-3329, 2020.

[5] S. Saravanan and P. Sudhakar, "Analysis of mobile internet speed, signal strength and FMDH antenna design for improved internet speed," The Journal of Supercomputing, vol. 6, pp. 1-27, 2018.

[6] A. K. Sangaiah, D. V. Medhane, T. Han, M. S. Hossain, and G. Muhammad, "Enforcing position-based confidentiality with machine learning paradigm through mobile edge computing in real-time industrial informatics," IEEE Transactions on Industrial Informatics, vol. 15, no. 7, pp. 4189-4196, 2019.

[7] L.-P. Song, Z. Jin, G.-Q. Sun, J. Zhang, and X. Han, "Influence of removable devices on computer worms: dynamic analysis and control strategies," Computers \& Mathematics with Applications, vol. 61, no. 7, pp. 1823-1829, 2011.

[8] H. Berghel, "The code red worm," Communications of the $A C M$, vol. 44, no. 12, pp. 15-19, 2001.

[9] C. Shannon and D. Moore, "The spread of the witty worm," IEEE Security \& Privacy, vol. 2, no. 4, pp. 46-50, 2004.

[10] P. Jungck and S. S. Y. Shim, "Issues in high-speed internet security," Computer, vol. 37, no. 7, pp. 36-42, 2004.

[11] J. O. Kephart and S. R. White, "Directed-graph epidemiological models of computer viruses," in Proceedings of the IEEE Computer Society Symposium on Research in Security and Privacy, pp. 343-359, IEEE Computer Society Press, Oakland, CA, USA, May 1991.

[12] J. C. Martin, L. L. Burge III, J. I. Gill, A. N. Washington, and M. Alfred, "Modelling the spread of mobile malware," International Journal of Computer Aided Engineering and Technology, vol. 2, no. 1, pp. 3-14, 2010.

[13] L. Feng, X. Liao, Q. Han, and H. Li, "Dynamical analysis and control strategies on malware propagation model," Applied Mathematical Modelling, vol. 37, no. 16-17, pp. 8225-8236, 2013.

[14] M. T. Signes-Pont, A. Cortés-Castillo, H. Mora-Mora, and J. Szymanski, "Modelling the malware propagation in mobile computer devices," Computers \& Security, vol. 79, no. 2, pp. 80-93, 2018.

[15] W. Xia, Z.-H. Li, Z.-Q. Chen, and Z.-Z. Yuan, "Commwarrior worm propagation model for smart phone networks," The
Journal of China Universities of Posts and Telecommunications, vol. 15, no. 2, pp. 60-66, 2018.

[16] J. Jia and X. Jing, "Stability analysis of a disease resistance SEIRS model with nonlinear incidence rate," Advances in Difference Equations, vol. 1, no. 75, pp. 1-13, 2018.

[17] C. C. Zou, W. Gong, and D. Towsley, "Worm propagation modeling and analysis under dynamic quarantine defense," in Proceedings of the 2003 ACM Workshop on Rapid Malcode (WORM'03), pp. 51-60, Washington, DC, USA, October 2003.

[18] J. Guan, J. Li, and Z. Jiang, "The design and implementation of a multidimensional and hierarchical web anomaly detection system," Intelligent Automation and Soft Computing, vol. 5, p. 1, 2018.

[19] X. B. Liu and L. J. Yang, "Stability analysis of an SEIQV epidemic model with saturated incidence rate," Nonlinear Analysis: Real World Applications, vol. 13, no. 6, pp. 26712679, 2012.

[20] Z. Zhang, S. Kundu, and R. Wei, "A delayed epidemic model for propagation of malicious codes in wireless sensor network," Mathematics, vol. 7, no. 5, pp. 1-18, 2019.

[21] X. Xiao, P. Fu, C. Dou, Q. Li, G. Hu, and S. Xia, "Design and analysis of SEIQR worm propagation model in mobile internet," Communications in Nonlinear Science and Numerical Simulation, vol. 43, pp. 341-350, 2017.

[22] B. K. Mishra and N. Jha, "SEIQRS model for the transmission of malicious objects in computer network," Applied Mathematical Modelling, vol. 34, no. 3, pp. 710-715, 2010.

[23] F. Wang, Y. Zhang, C. Wang, J. Ma, and S. Moon, "Stability analysis of a SEIQV epidemic model for rapid spreading worms," Computers \& Security, vol. 29, no. 4, pp. 410-418, 2010.

[24] X. Wang, H. Peng, B. Shi, D. Jiang, S. Zhang, and B. Chen, "Optimal vaccination strategy of a constrained time-varying SEIR epidemic model," Communications in Nonlinear Science and Numerical Simulation, vol. 67, pp. 37-48, 2019.

[25] B. K. Mishra and S. K. Pandey, "Dynamic model of worm propagation in computer network," Applied Mathematical Modelling, vol. 38, no. 7-8, pp. 2173-2179, 2014.

[26] D. R. Merkin, Introduction to the Theory of Stability, Springer, New York, NY, USA, 1997.

[27] R. C. Robinson, An Introduction to Dynamical Systems: Continuous and Discrete, Prentice-Hall, Upper Saddle River, NJ, USA, 2004.

[28] D. Cheng, J. Wang, and X. HU, “An extension of LaSalle's invariabce principle and its application tomulti-agent consensus," IEEE Transaction on Automatic Control, vol. 53, no. 7, pp. 765-1770, 2008. 